\title{
THE ROLE OF SURGERY IN THE TREATMENT OF MALIGNANT TUMOURS OF THE SPINE
}

\author{
N. S. Martin and Jean Williamson, Belfast, Northern Ireland
}

From the Northern Ireland Orthopaedic Service, Belfast

\begin{abstract}
Malignant tumours of the spine present special problems in management because of the danger of spinal cord involvement. In these cases operation may have a double role, in diagnosis and in treatment. There is in the literature little mention of lateral approaches in the treatment of such lesions, so an account of our experience may prove of interest. Whatever influence radiotherapy and chemotherapy may exert on the course of the disease, operation is often of value in the relief of symptoms. Halnan and Roberts (1967) observed that energetic treatment of patients with spinal metastases is often considered to be meddlesome. Nevertheless if it is possible to offer relief for the more distressing symptoms by surgical means, this should not be neglected simply because the results will probably be short-term.

A review of this small series reveals a few gratifying results which might produce a cautious optimism in planning the management of patients presenting with a solitary spinal lesion.
\end{abstract}

\section{MATERIAL}

The series consists of nineteen patients with solitary vertebral lesions shown by biopsy to be malignant. Since they presented similar clinical features and problems due to the location of the tumour, irrespective of its nature, it is expedient to consider them as a group. The similarity of clinical pattern and progress in such cases was stressed by Brice and McKissock (1965).

All available notes and radiographs have been studied, and with one exception all survivors have been examined personally. The slides have been reviewed by the pathologists whose reports are included. Special attention has been given to searching, with hindsight, for any aspect of the material which would have been valuable in prognosis.

\section{RESULTS}

The findings are summarised in Tables I to III. We have measured the results in terms of absolute and relative success. Long-term survival is the ideal, but preservation of limb function and the relief of pain even for a few months are worth while. The results of treatment are summarised in Table IV.

With one exception, all patients in this series in whom pain was not relieved and neurological function was not improved died within four months (Cases 1, 2, 3, 7, 13, 19); the patient in Case 6 survived for sixteen months. Ten patients survived for a year or longer, and of these all except one (Case 6) derived material benefit from treatment (Cases 4, 8, 9, 10, 14, 15, 16, 17, 18). Two patients (Cases 5 and 12) were improved symptomatically although they survived for less than a year.

\section{INDICATION FOR OPERATION}

1) When clinical and radiological investigations have failed to establish a diagnosis or reveal a primary lesion the diagnosis must be reached without further delay (Cases 1, 4, 8, 9, $11,13,14)$. Other workers have mentioned the danger of wasting time on extensive clinical searches for an elusive primary tumour (Mullan and Evans 1957, Brice and McKissock 1965, 
TABLE I

Metastatic Carcinoma (Eight Cases)

\begin{tabular}{|c|c|c|c|c|c|c|c|}
\hline $\begin{array}{c}\text { Case } \\
\text { number }\end{array}$ & Sex & $\begin{array}{c}\text { Age } \\
\text { (years) }\end{array}$ & $\begin{array}{l}\text { Vertebrae } \\
\text { afiected }\end{array}$ & First symptoms & $\begin{array}{l}\text { Neurological } \\
\text { involvement }\end{array}$ & Investigations & $\begin{array}{l}\text { Radiological } \\
\text { features }\end{array}$ \\
\hline 1 & Male & 50 & C.5 & $\begin{array}{l}\text { Upper thoracic } \\
\text { pain } 2 \text { months }\end{array}$ & Root pain & $\begin{array}{l}\text { Pyelography, sig- } \\
\text { moidoscopy and } \\
\text { examination of } \\
\text { sputum negative. } \\
\text { No Bence-Jones } \\
\text { protein }\end{array}$ & $\begin{array}{l}\text { Suggestive of } \\
\text { secondary } \\
\text { deposits }\end{array}$ \\
\hline 2 & Female & 68 & T.3 & $\begin{array}{l}\text { Girdle pain } \\
3 \text { months }\end{array}$ & $\begin{array}{l}\text { Sudden paraplegia } \\
\text { with sphincter } \\
\text { involvement, and } \\
\text { slight sensory loss }\end{array}$ & Tomography & $\begin{array}{c}\text { Destructive } \\
\text { vertebral lesion }\end{array}$ \\
\hline 3 & Male & 73 & $\begin{array}{l}\text { T.8, } 9 \\
\text { and } 10\end{array}$ & $\begin{array}{l}\text { Lower thoracic } \\
\text { pain } 7 \text { months }\end{array}$ & $\begin{array}{c}\text { Complete motor } \\
\text { and sensory } \\
\text { paraplegia with } \\
\text { sphincter } \\
\text { involvement }\end{array}$ & Not recorded & $\begin{array}{c}\text { Destructive } \\
\text { lesion with } \\
\text { paravertebral } \\
\text { shadow }\end{array}$ \\
\hline 4 & Male & 60 & L.1 & $\begin{array}{l}\text { Pain left hip } \\
\text { and groin. } \\
\text { Pain on } \\
\text { micturition }\end{array}$ & Root pain & $\begin{array}{l}\text { Pyelography and } \\
\text { cystoscopy } \\
\text { negative. } \\
\text { Normal acid } \\
\text { and alkaline } \\
\text { phosphatase }\end{array}$ & $\begin{array}{l}\text { 1) Moderate } \\
\text { degeneration } \\
\text { lumbar spine. } \\
\text { 2) Four months } \\
\text { later, lytic lesion } \\
\text { in L.1 }\end{array}$ \\
\hline 5 & Male & 52 & L. 5 & $\begin{array}{l}\text { Low back pain } \\
\text { and right sciatica }\end{array}$ & Root pain & \begin{tabular}{|} 
Electrophoresis \\
of serum protein. \\
Acid and alkaline \\
phosphatase: \\
findings \\
inconclusive
\end{tabular} & $\begin{array}{l}\text { Almost complete } \\
\text { disappearance } \\
\text { of } \mathrm{L} .5\end{array}$ \\
\hline 6 & Male & 45 & $\mathrm{~T} .7$ & $\begin{array}{c}\text { Dorsal pain } \\
1 \text { year }\end{array}$ & $\begin{array}{l}\text { Diminished power } \\
\text { in legs progressing } \\
\text { to complete motor } \\
\text { and sensory } \\
\text { paraplegia }\end{array}$ & Not recorded & $\begin{array}{l}\text { Destructive } \\
\text { changes T.7 with } \\
\text { paravertebral } \\
\text { soft-tissue } \\
\text { swelling }\end{array}$ \\
\hline 7 & Female & 63 & L. 5 & $\begin{array}{l}\text { Bilateral } \\
\text { sciatica } \\
6 \text { months }\end{array}$ & $\begin{array}{c}\text { Cauda equina } \\
\text { lesion }\end{array}$ & Not recorded & $\begin{array}{l}\text { Radiographs not } \\
\text { available, but } \\
\text { reported as } \\
\text { suggestive of } \\
\text { tuberculosis }\end{array}$ \\
\hline 8 & Female & 58 & T.11 & $\begin{array}{l}\text { Back pain } \\
\text { radiating to } \\
\text { both legs } \\
9 \text { months }\end{array}$ & $\begin{array}{c}\text { Paraplegia with } \\
\text { sphincter involve- } \\
\text { ment. Sensation } \\
\text { preserved. No } \\
\text { pain after onset } \\
\text { of paraplegia }\end{array}$ & $\begin{array}{l}\text { Intravenous } \\
\text { pyelograph; } \\
\text { electrophoresis } \\
\text { of serum } \\
\text { proteins: } \\
\text { normal results }\end{array}$ & $\begin{array}{l}\text { Destructive } \\
\text { lesion T.11 }\end{array}$ \\
\hline
\end{tabular}

Halnan and Roberts 1967). Valderrama and Bullough (1968) noted negative results in BenceJones protein and electrophoresis studies in cases of myeloma.

2) Surgical decompression will often be required when there is neurological deterioration. Decompression has sometimes been effected by radiotherapy when the lesion is radiosensitive but operation is probably more expeditious and effective.

3) In an extensively infiltrated vertebra there is an increased likelihood of pathological collapse (Cases 10, 12, 14, 16, 17). Mullan and Evans (1957) reported cases of pathological fracture. Local excision with grafting when necessary, followed by radiotherapy, is the logical method of reducing this risk. 
TABLE I-continued

Metastatic Carcinoma (Eight Cases)

\begin{tabular}{|c|c|c|c|c|c|c|}
\hline $\begin{array}{c}\text { Macroscopic } \\
\text { appearance } \\
\text { at operation }\end{array}$ & $\begin{array}{l}\text { Site of } \\
\text { primary } \\
\text { tumour }\end{array}$ & Treatment & $\begin{array}{c}\text { Neurological } \\
\text { function after } \\
\text { operation }\end{array}$ & Pain & Survival & $\begin{array}{l}\text { Special } \\
\text { features }\end{array}$ \\
\hline $\begin{array}{l}\text { Body of C.5 } \\
\text { replaced by } \\
\text { greyish tumour }\end{array}$ & Lung & $\begin{array}{l}\text { Curettage (biopsy) } \\
\text { by anterior approach: } \\
\text { Kiel bone graft. } \\
\text { Radiotherapy }\end{array}$ & $\begin{array}{l}\text { Deteriorated. } \\
\text { Progressive } \\
\text { weakening } \\
\text { of arms }\end{array}$ & $\begin{array}{l}\text { Not } \\
\text { relieved }\end{array}$ & $\begin{array}{c}\text { Died } 4 \text { months } \\
\text { after } \\
\text { operation }\end{array} \mid$ & $\begin{array}{c}\text { Primary lesion } \\
\text { not identified } \\
\text { before } \\
\text { operation }\end{array}$ \\
\hline $\begin{array}{l}\text { Resembles } \\
\text { caries sicca }\end{array}$ & Lung & $\begin{array}{l}\text { Curettage (biopsy). } \\
\text { Postero-lateral } \\
\text { decompression }\end{array}$ & Unchanged & $\begin{array}{c}\text { Not a } \\
\text { feature }\end{array}$ & $\begin{array}{c}\text { Died } 2 \text { weeks } \\
\text { after operation } \\
\text { from pulmonary } \\
\text { embolism }\end{array}$ & $\begin{array}{c}\text { Diagnosis } \\
\text { established } \\
\text { by biopsy }\end{array}$ \\
\hline $\begin{array}{l}\text { Appearance of } \\
\text { lesion not } \\
\text { recorded. } \\
\text { Dura hard and } \\
\text { not pulsating }\end{array}$ & Prostate & $\begin{array}{c}\text { Attempted } \\
\text { decompression }\end{array}$ & Unchanged & $\begin{array}{c}\text { Not a } \\
\text { feature }\end{array}$ & $\begin{array}{c}\text { Died } 2 \text { months } \\
\text { after } \\
\text { operation }\end{array}$ & $\begin{array}{c}\text { Primary lesion } \\
\text { not identified } \\
\text { before } \\
\text { operation }\end{array}$ \\
\hline Not recorded & Prostate & $\begin{array}{l}\text { Curettage (biopsy) } \\
\text { by renal approach. } \\
\text { Iliac and Kiel bone } \\
\text { graft. Radiotherapy }\end{array}$ & $\begin{array}{c}\text { Remained } \\
\text { unimpaired }\end{array}$ & Relieved & \begin{tabular}{|} 
Died 20 months \\
after \\
operation
\end{tabular} \mid & $\begin{array}{c}\text { Primary lesion } \\
\text { not indentified } \\
\text { before } \\
\text { operation }\end{array}$ \\
\hline $\begin{array}{c}\text { Rubbery } \\
\text { vascular lesion }\end{array}$ & Kidney & $\begin{array}{c}\text { Curettage (biopsy) } \\
\text { by transperitoneal } \\
\text { approach. } \\
\text { Autogenous graft. } \\
\text { Radiotherapy }\end{array}$ & $\begin{array}{l}\text { Remained } \\
\text { unimpaired }\end{array}$ & Relieved & $\begin{array}{l}\text { Died } 8 \text { months } \\
\text { after } \\
\text { operation }\end{array}$ & $\begin{array}{l}\text { Diagnosis } \\
\text { established } \\
\text { by biopsy }\end{array}$ \\
\hline $\begin{array}{l}\text { No pus in the } \\
\text { paravertebral } \\
\text { swelling }\end{array}$ & Kidney & $\begin{array}{l}\text { Curettage (biopsy). } \\
\text { Autogenous graft. } \\
\text { Radiotherapy }\end{array}$ & Unchanged & $\begin{array}{l}\text { Not } \\
\text { relieved }\end{array}$ & $\begin{array}{c}\text { Died } 16 \text { months } \\
\text { after } \\
\text { operation }\end{array}$ & \\
\hline Not recorded & ? Ovary & $\begin{array}{l}\text { Curettage (biopsy) } \\
\text { by renal approach. } \\
\text { L. } 4 / 5 \text { nerve roots } \\
\text { freed. Radiotherapy }\end{array}$ & Unchanged & $\begin{array}{l}\text { Not } \\
\text { relieved }\end{array}$ & $\begin{array}{c}\text { Died } 3 \text { months } \\
\text { after } \\
\text { operation }\end{array}$ & $\begin{array}{c}\text { Primary lesion } \\
\text { not identified } \\
\text { before } \\
\text { operation }\end{array}$ \\
\hline $\begin{array}{l}\text { Infiltration } \\
\text { of entire body } \\
\text { of } \mathrm{T} .11\end{array}$ & $\begin{array}{l}\text { Thyroid } \\
\text { gland }\end{array}$ & $\begin{array}{l}\text { Curettage (biopsy). } \\
\text { Postero-lateral } \\
\text { decompression. } \\
\text { Radiotherapy to spine } \\
\text { followed by } \\
\text { I }^{131} \text { therapy }\end{array}$ & Recovered & $\begin{array}{c}\text { Not a } \\
\text { feature }\end{array}$ & $\begin{array}{c}\text { Still alive } \\
\text { and well } \\
5 \frac{1}{2} \text { years after } \\
\text { operation }\end{array}$ & $\begin{array}{c}\text { Primary lesion } \\
\text { not identified } \\
\text { before } \\
\text { operation }\end{array}$ \\
\hline
\end{tabular}

\section{TECHNIQUES OF OPERATION}

Most published series of malignant spinal tumours are from neurosurgical centres where the main surgical procedure has been a posterior approach by laminectomy with the object of decompressing the cord or cauda equina and, if possible, obtaining material on which to base a diagnosis.

We believe that laminectomy has a restricted use. By such an approach it is possible to expose only the posterior half of the cord and spinal canal. As observed by others (Brice and McKissock 1965) many of these tumours are anteriorly placed or more commonly form an encircling cuff.

VOL. 52 B, NO. 2, MAY 1970 
TABLE II

Reticuloses (Seven Cases)

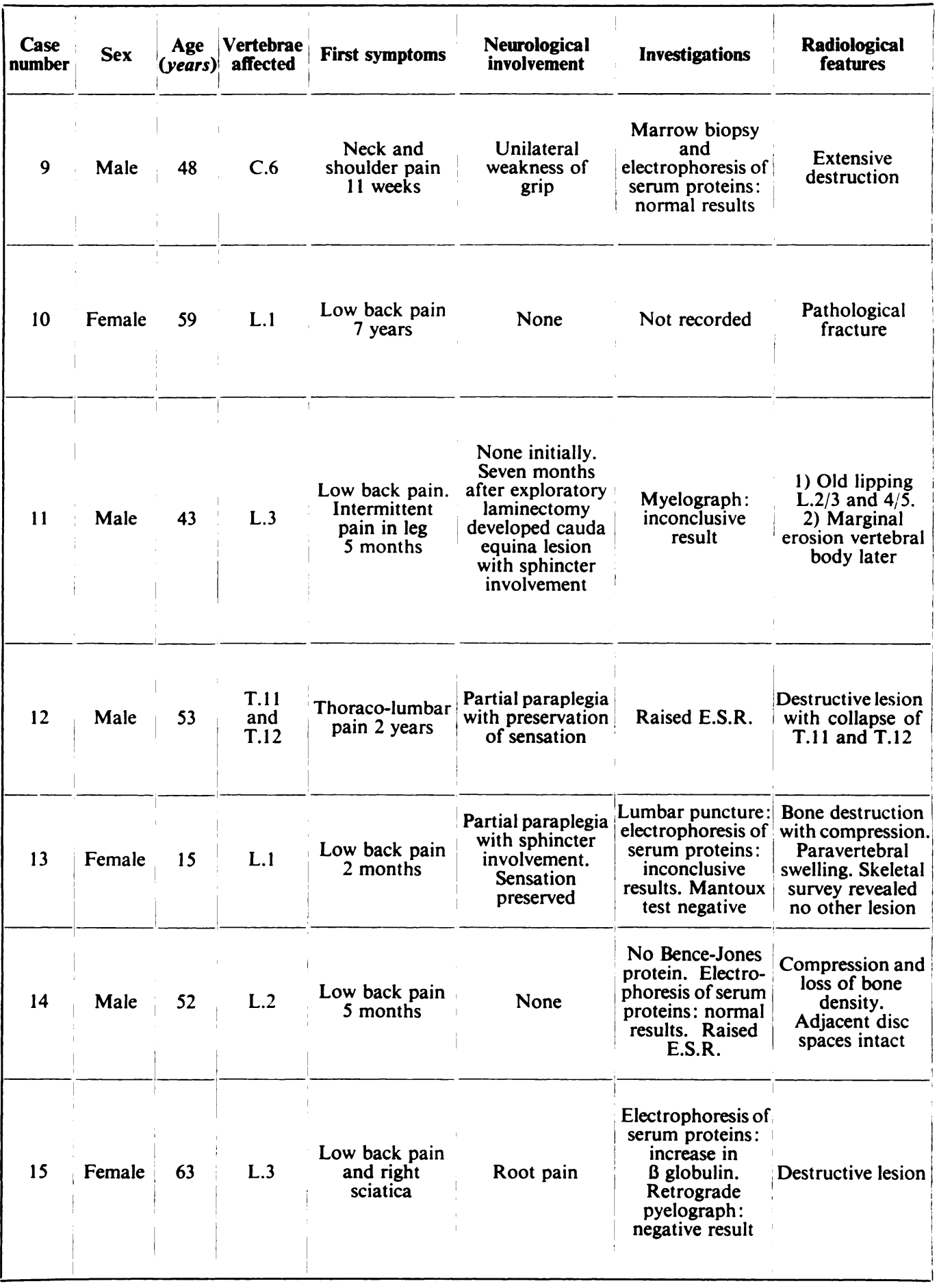


TABLE II-continued

Reticuloses (Seven Cases)

\begin{tabular}{|c|c|c|c|c|c|c|}
\hline $\begin{array}{l}\text { Macroscopic } \\
\text { appearance } \\
\text { at operation }\end{array}$ & Histology & Treatment & $\begin{array}{c}\text { Neurological } \\
\text { function after } \\
\text { operation }\end{array}$ & Pain & Survival & Special features \\
\hline $\begin{array}{l}\text { Body of C. } 6 \\
\text { replaced by } \\
\text { tumour }\end{array}$ & Myeloma & $\begin{array}{l}\text { Curettage } \\
\text { (biopsy) by } \\
\text { anterior } \\
\text { approach. } \\
\text { Autogenous } \\
\text { graft. } \\
\text { Radiotherapy }\end{array}$ & Recovered & $\begin{array}{l}\text { Good } \\
\text { relief }\end{array}$ & \begin{tabular}{|c|} 
Alive and \\
comfortable \\
$3 \frac{1}{2}$ years after \\
operation. \\
Deposits in ribs
\end{tabular} & $\begin{array}{l}\text { Clinical investiga- } \\
\text { tions did not pro- } \\
\text { vide diagnosis. } \\
\text { Marrow puncture } \\
\text { showed normal } \\
\text { pattern } 2 \text { years } \\
\text { after operation }\end{array}$ \\
\hline $\begin{array}{l}\text { Body of L.1 } \\
\text { a collapsed } \\
\text { shell }\end{array}$ & Myeloma & $\begin{array}{c}\text { Curettage } \\
\text { (biopsy) by } \\
\text { extraperitoneal } \\
\text { approach. } \\
\text { Autogenous } \\
\text { graft. } \\
\text { Radiotherapy }\end{array}$ & $\begin{array}{l}\text { Remained } \\
\text { unimpaired }\end{array}$ & $\begin{array}{l}\text { Completely } \\
\text { relieved }\end{array}$ & $\begin{array}{c}\text { Alive and well } \\
12 \text { years after } \\
\text { operation. } \\
\text { Deposits in } \\
\text { skull }\end{array}$ & $\begin{array}{l}\text { Provisionally } \\
\text { diagnosed and } \\
\text { irradiated as } \\
\text { osteoclastoma }\end{array}$ \\
\hline $\begin{array}{l}\text { 1) At laminectomy } \\
\text { nothing abnormal } \\
\text { found. } \\
\text { 2) Seven months } \\
\text { later postero- } \\
\text { lateral decom- } \\
\text { pression by renal } \\
\text { approach: body of } \\
\text { L. } 3 \text { collapsed and } \\
\text { invaded by } \\
\text { tumour. Cauda } \\
\text { equina encircled }\end{array}$ & $\begin{array}{l}\text { Malignant } \\
\text { reticulosis }\end{array}$ & $\begin{array}{c}\text { Curettage } \\
\text { (biopsy). } \\
\text { Radiotherapy }\end{array}$ & $\begin{array}{l}\text { Fair } \\
\text { recovery } \\
\text { of power } \\
\text { but not of } \\
\text { sphincter } \\
\text { control }\end{array}$ & $\begin{array}{l}\text { Moderate } \\
\text { relief }\end{array}$ & $\begin{array}{c}\text { Alive } 6 \text { months } \\
\text { after second } \\
\text { operation but } \\
\text { little } \\
\text { improvement }\end{array}$ & $\begin{array}{l}\text { Initial radio- } \\
\text { graphs and } \\
\text { laminectomy } \\
\text { failed to reveal } \\
\text { presence of } \\
\text { vertebral tumour } \\
\text { which became } \\
\text { manifest by } \\
\text { neurological } \\
\text { deterioration }\end{array}$ \\
\hline $\begin{array}{l}\text { Unstable spine. } \\
\text { No pus, but } \\
\text { appearance of } \\
\text { granulation } \\
\text { tissue in } \\
\text { paravertebral } \\
\text { abscess }\end{array}$ & $\begin{array}{l}\text { Reticulum } \\
\text { cell } \\
\text { tumour }\end{array}$ & $\begin{array}{l}\text { Curettage } \\
\text { (biopsy) by } \\
\text { transpleural } \\
\text { approach. } \\
\text { Autogenous } \\
\text { graft. } \\
\text { Radiotherapy }\end{array}$ & $\begin{array}{l}\text { Recovery of } \\
\text { paralysis. } \\
\text { Slight girdle } \\
\text { pain } \\
\text { recurred } \\
\text { after } \\
5 \text { months }\end{array}$ & $\begin{array}{c}\text { Relieved } \\
\text { for several } \\
\text { months }\end{array}$ & $\begin{array}{c}\text { Died } 9 \text { months } \\
\text { after } \\
\text { operation }\end{array}$ & \\
\hline $\begin{array}{l}\text { Psoas } \\
\text { contained } \\
\text { thick pus }\end{array}$ & $\begin{array}{l}\text { Reticulum } \\
\text { cell } \\
\text { sarcoma }\end{array}$ & $\begin{array}{l}\text { Postero-lateral } \\
\text { decompression. } \\
\text { Curettage } \\
\text { (biopsy). } \\
\text { Radiotherapy }\end{array}$ & $\begin{array}{l}\text { Partial } \\
\text { recovery }\end{array}$ & $\begin{array}{l}\text { Not } \\
\text { relieved }\end{array}$ & $\begin{array}{c}\text { Died } 3 \text { months } \\
\text { after } \\
\text { operation }\end{array}$ & \\
\hline $\begin{array}{l}\text { L.2 replaced by } \\
\text { rubbery } \\
\text { tumour }\end{array}$ & $\begin{array}{l}\text { First report } \\
\text { reticulum } \\
\text { cell tumour. } \\
\text { Three months } \\
\text { later biopsy } \\
\text { showed } \\
\text { myelomatosis }\end{array}$ & $\begin{array}{c}\text { Curettage } \\
\text { (biopsy) by } \\
\text { extraperitoneal } \\
\text { approach. } \\
\text { Autogenous } \\
\text { graft. } \\
\text { Radiotherapy }\end{array}$ & $\begin{array}{c}\text { Remained } \\
\text { unimpaired }\end{array}$ & $\begin{array}{l}\text { Relieved } \\
\text { for } 1 \text { year }\end{array}$ & $\begin{array}{c}\text { Within } \\
15 \text { months had } \\
\text { multiple } \\
\text { deposits. Died } \\
2 \text { years } 2 \text { months } \\
\text { after } \\
\text { operation }\end{array}$ & \\
\hline Not recorded & $\begin{array}{c}\text { First reported } \\
\text { as anaplastic } \\
\text { carcinoma. } \\
\text { Four years } \\
\text { later revised } \\
\text { report } \\
\text { myeloma }\end{array}$ & $\begin{array}{c}\text { Curettage } \\
\text { (biopsy). } \\
\text { Radiotherapy }\end{array}$ & $\begin{array}{c}\text { Remained } \\
\text { unimpaired }\end{array}$ & $\begin{array}{l}\text { Completely } \\
\text { relieved }\end{array}$ & $\begin{array}{l}\text { Remained well } \\
\text { and active until } \\
\text { death } 4 \text { years } \\
\text { after operation } \\
\text { from myocardial } \\
\text { infarction. A } \\
\text { few deposits } \\
\text { suspected in } \\
\text { thoracic spine }\end{array}$ & $\begin{array}{c}\text { Atypical } \\
\text { appearance of } \\
\text { biopsy material }\end{array}$ \\
\hline
\end{tabular}

VOL. 52 B, NO. 2, MAY 1970 
TABLE III

local Primary Tumours (Four Cases)

\begin{tabular}{|c|c|c|c|c|c|c|c|}
\hline $\begin{array}{c}\text { Case } \\
\text { number }\end{array}$ & Sex & $\begin{array}{c}\text { Age } \\
\text { (years) }\end{array}$ & $\begin{array}{l}\text { Vertebrae } \\
\text { affected }\end{array}$ & First symptoms & $\begin{array}{c}\text { Neurological } \\
\text { involvement }\end{array}$ & Investigations & $\begin{array}{c}\text { Radiological } \\
\text { features }\end{array}$ \\
\hline 16 & Male & 17 & L. 1 & $\begin{array}{l}\text { Low back pain. } \\
\text { Recurrence } \\
\text { five years later }\end{array}$ & $\begin{array}{l}\text { None initially. } \\
\text { Five years later } \\
\text { partial paraplegia } \\
\text { with preservation } \\
\text { of sensation and } \\
\text { sphincter control }\end{array}$ & Serial E.S.R. & $\begin{array}{l}\text { First appearance } \\
\text { suggested tuber- } \\
\text { culosis of spine. } \\
\text { Recovery on anti- } \\
\text { tuberculous } \\
\text { therapy. } \\
\text { Five years later } \\
\text { wedging L.1 }\end{array}$ \\
\hline 17 & Male & 12 & L.4 & $\begin{array}{l}\text { Low back pain } \\
\text { and severe } \\
\text { kyphoscoliosis }\end{array}$ & None & $\begin{array}{l}\text { Mantoux test } \\
\text { negative. } \\
\text { E.S.R. normal }\end{array}$ & $\begin{array}{l}\text { Flattening and } \\
\text { lateral angulation } \\
\text { of L.4 }\end{array}$ \\
\hline 18 & Female & 18 & Sacrum & $\begin{array}{l}\text { Sciatica. } \\
\text { Local swelling } \\
\text { obstructed } \\
\text { labour. } \\
\text { Intolerable } \\
\text { local pain }\end{array}$ & $\begin{array}{l}\text { Unilateral } \\
\text { paralysis of all } \\
\text { muscle groups } \\
\text { below the knee } \\
\text { and sphincter } \\
\text { involvement }\end{array}$ & Needle biopsy & $\begin{array}{l}\text { Destructive } \\
\text { lesion left side } \\
\text { of sacrum }\end{array}$ \\
\hline 19 & Female & 20 & Sacrum & $\begin{array}{l}\text { Pain in right } \\
\text { hip and heel }\end{array}$ & $\begin{array}{l}\text { Unilateral } \\
\text { foot drop. } \\
\text { Sphincter } \\
\text { involvement }\end{array}$ & E.S.R. raised & $\begin{array}{l}\text { Destructive } \\
\text { right side } \\
\text { sacrum- } \\
\text { part of ilium }\end{array}$ \\
\hline
\end{tabular}

In 1954 Capener observed that lateral rhachotomy, which he developed for the treatment of Pott's paraplegia, could be employed in a variety of conditions including spinal tumours. Experience here supports this view: in this centre spinal lesions are often referred to surgeons whose interest in spinal surgery has been stimulated by their work on spinal tuberculosis. Direct focal procedures as used in tuberculous lesions of the spine were employed in seventeen of the nineteen patients in this series. Of two patients with sacral tumours (Cases 18 and 19) one underwent a two-stage removal through abdominal and posterior approaches.

While we admit that the series is small, we are encouraged by the results of frontal attacks on such lesions. Direct focal operation provides the surgeon with an opportunity of seeing the lesion in situ and of determining its size and extent. It has been noted that radiographic changes tend to underestimate the real extent of pathological destruction (Young and Funk 1953). In the thoracic spine, because of the narrowness of the vertebral canal, it is quite impossible by laminectomy to carry out proper inspection of the area in front of the dura without extensive removal of one or more articular processes. Indeed it is usually necessary to divide two or more nerve roots before the cord can be rotated sufficiently to allow even a glimpse of its anterior aspect. Apart from surgical excision of lesions across the thoracic and abdominal cavities, it is possible by a posterior extension of such incisions to perform lateral decompression of the cord or cauda equina. In some patients, after excision of the tumour material spinal grafting has been carried out to improve stability, thereby avoiding subsequent angulation which might imperil the cord (Cases 1, 2, 4, 6, 9, 10, 14, 17).

Although the Craig vertebral biopsy needle has been used as a diagnostic aid, this was found to be an unreliable method of obtaining biopsy material and is now seldom employed. Furthermore, inspection at operation has shown that tumours may be walled in by much 
TABLE III-continued

local Primary Tumours (Four Cases)

\begin{tabular}{|c|c|c|c|c|c|c|}
\hline $\begin{array}{l}\text { Macroscopic } \\
\text { appearance } \\
\text { at operation }\end{array}$ & Histology & Treatment & $\begin{array}{c}\text { Neurological } \\
\text { function after } \\
\text { operation }\end{array}$ & Pain & Survival & Special features \\
\hline $\begin{array}{c}\text { Fleshy } \\
\text { tumour } \\
\text { invading } \\
\text { psoas and } \\
\text { dura }\end{array}$ & $\begin{array}{l}\text { Doubtful. } \\
\text { ?Fibrosarcoma. } \\
\begin{array}{c}\text { ? Hodgkin's } \\
\text { disease }\end{array}\end{array}$ & $\begin{array}{c}\text { Curettage } \\
\text { (biopsy). } \\
\text { Postero-lateral } \\
\text { decompression. } \\
\text { Radiotherapy }\end{array}$ & Recovered & $\begin{array}{c}\text { Completely } \\
\text { relieved }\end{array}$ & $\begin{array}{l}\text { Alive and well } \\
10 \text { years after } \\
\text { operation }\end{array}$ & $\begin{array}{c}\text { Provisional } \\
\text { diagnosis } \\
\text { tuberculosis of } \\
\text { spine and treated } \\
\text { successfully as } \\
\text { such for } 2 \text { years }\end{array}$ \\
\hline$\underset{\substack{\text { Dry } \\
\text { semi-caseous } \\
\text { material }}}{\mid}$ & $\begin{array}{l}\text { Chondro- } \\
\text { sarcoma }\end{array}$ & $\begin{array}{c}\text { Curettage (biopsy) } \\
\text { by extraperitoneal } \\
\text { approach. Correc- } \\
\text { tion of deformity } \\
\text { and autogenous } \\
\text { graft }\end{array}$ & $\begin{array}{c}\text { Remained } \\
\text { unimpaired }\end{array}$ & $\begin{array}{c}\text { Completely } \\
\text { relieved }\end{array}$ & $\begin{array}{l}\text { Alive and well } \\
11 \text { years after } \\
\text { operation }\end{array}$ & \\
\hline $\begin{array}{l}420 \text { gramme } \\
\text { tumour }\end{array}$ & $\begin{array}{l}\text { 1) Aspiration } \\
\text { biopsy report } \\
\text { osteoclastoma. } \\
\text { 2) Surgical } \\
\text { biopsy report } \\
\text { osteogenic } \\
\text { sarcoma }\end{array}$ & $\begin{array}{l}\text { Cordotomy. Two- } \\
\text { stage removal of } \\
\text { tumour by } \\
\text { abdominal and } \\
\text { posterior } \\
\text { approaches. } \\
\text { Radiotherapy }\end{array}$ & $\begin{array}{l}\text { Complete } \\
\text { recovery of } \\
\text { sphincter } \\
\text { control } \\
\text { otherwise } \\
\text { unchanged }\end{array}$ & $\begin{array}{l}\text { Completely } \\
\text { relieved }\end{array}$ & $\begin{array}{l}\text { Alive and well } \\
2 \frac{1}{2} \text { years after } \\
\text { operation }\end{array}$ & $\begin{array}{l}\text { The discrepancies } \\
\text { between the } \\
\text { pathology reports } \\
\text { may be due to the } \\
\text { influence of radio- } \\
\text { therapy before } \\
\text { the surgical re- } \\
\text { moval and biopsy }\end{array}$ \\
\hline $\begin{array}{l}\text { Highly } \\
\text { vascular }\end{array}$ & $\begin{array}{c}\text { Anaplastic } \\
\text { highly } \\
\text { invasive } \\
\text { embryoma }\end{array}$ & $\begin{array}{l}\text { Removal } \\
\text { incomplete due } \\
\text { to torrential } \\
\text { haemorrhage }\end{array}$ & Unchanged & Unrelieved & $\begin{array}{l}\text { Died } 48 \text { hours } \\
\text { after operation }\end{array}$ & \\
\hline
\end{tabular}

cortical bone which would render difficult or impossible the acquisition of adequate representative material by paracentesis. By a direct approach tumour tissue can always be obtained.

\section{ILLUSTRATIVE CASES}

Case 8-A fifty-eight-year-old woman had for nine months had back pain radiating into both legs. For three months she had been paraplegic, with sphincter involvement but with preservation of sensation. She was admitted in July 1963. Radiographs showed a destructive lesion of the eleventh thoracic vertebra. Preliminary investigations failed to show definite evidence of a primary neoplasm. There was some enlargement of the left lobe of the thyroid gland: this had been present for many years and there had been no recent increase in size.

In August 1963 a postero-lateral decompression was done. The entire body of the eleventh vertebra was infiltrated with tumour, which displaced the theca backwards but did not invade it. Histological examination showed a well differentiated metastatic thyroid adenocarcinoma (Fig. 1). Sphincter control returned within a week of operation and motor power returned progressively in the following months.

Three weeks after operation, after tracer studies, the thyroid gland was irradiated with 100 millicuries of $\mathbf{I}^{131}$. The spine too was irradiated at the level of the lesion. In November 1963, after tracer studies had shown preferential uptake at the eleventh thoracic level, a further therapeutic dose of $\mathbf{I}^{\mathbf{1 3 1}}$ was administered. This was repeated in February and May 1964. Maintenance therapy by thyroxine was started.

Six years later the patient is reported to be well and active without local or metastatic recurrence

Case 16-A youth aged seventeen presented in 1951 with low back pain. Radiographs showed narrowing of the space between the twelfth thoracic and first lumbar vertebrae, with wedging of the latter vertebral body. A diagnosis of spinal tuberculosis was made, and the usual treatment with bed rest and drugs was given for two years. There was clinical and radiological recovery and the patient remained symptom-free with the lesion quiescent on serial radiographs for five years. In 1958 there

VOL. 52 B, NO. 2, MAY 1970 
was a recurrence of pain and a provisional diagnosis of reactivation of the lesion was made. He developed an almost complete transverse lesion of the cord. In January 1959 a postero-lateral decompression was done. The tumour resembled a secondary growth: it was fleshy and vascular and invading the psoas muscle without evidence of encapsulation. Complete removal was not possible. The histological appearances were consistent with a diagnosis of fibrosarcoma (Fig. 2).

One month after operation there was a striking return of power. Radiotherapy in a skin dose of 5,000 rads was administered. There has been persistent ulceration at the site of radiation: the dose given would now be considered excessive.

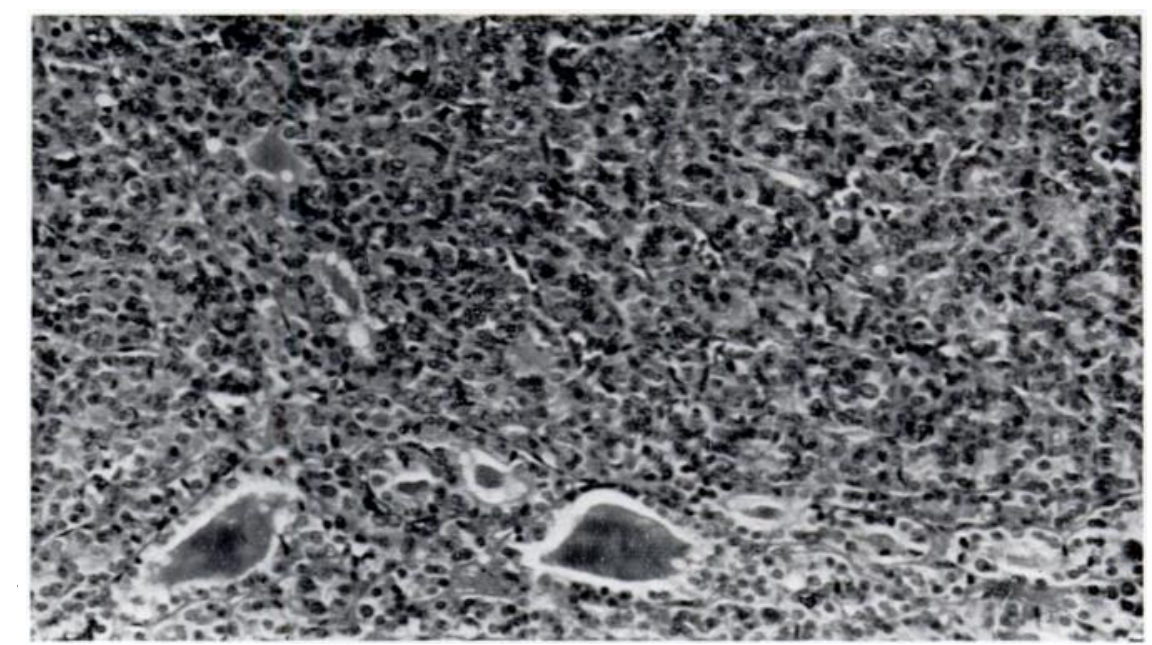

Fig. 1

A secondary carcinoma of the thyroid showing colloid in some acini. $(\times 180$.

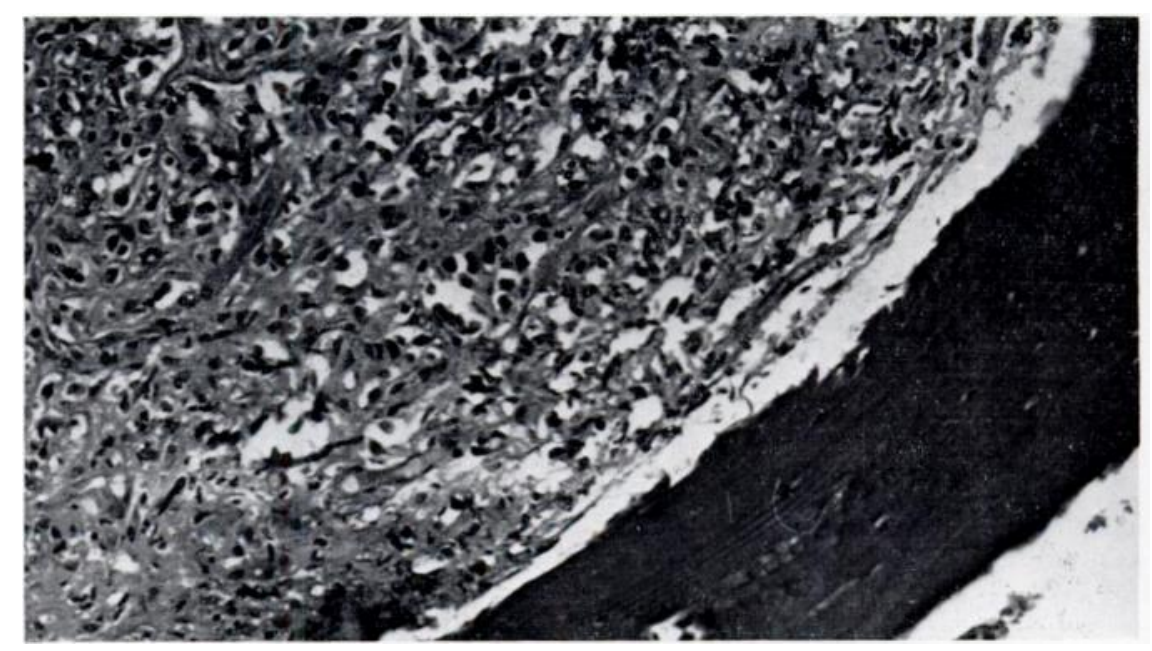

FIG. 2

Widespread infiltration of intertrabecular spaces by a somewhat pleomorphic tumour producing some collagen. $(\times 180$.

A few months later the biopsy material was reviewed and the possibility of Hodgkin's disease was considered although its rarity as a primary and solitary lesion in bone was stressed. Two pathologists still considered the lesion to be malignant. Four months after operation the patient had returned to light work. Since then he has gained weight and has remained in full employment for ten years without evidence of recurrence.

Case 10-A woman aged fifty-nine was seen in 1956. She had had low back pain for ten years. Serial radiographs showed a progressively destructive lesion in the first lumbar vertebra. This had already been diagnosed provisionally as an osteoclastoma and irradiated. The electrophoretic pattern of the 
serum proteins was normal. In February 1957 a pathological fracture of the vertebra occurred. The spine was exposed through a renal incision by an extraperitoneal approach: the body of the first and the upper half of the second lumbar vertebra were found to be almost totally replaced by bloodstained gelatinous tumour. After curettage the cavity was filled with autogenous iliac chips. Histological examination showed the appearances of myeloma (Fig. 3).

Five years later radiographs showed defects in the skull and electrophoresis showed almost a reversal of the albumin/globulin ratio. Nine years after operation there was radiological evidence of bone destruction in the sacrum. This area was accordingly irradiated. At that time BenceJones protein was not found in the urine and marrow biopsy showed moderately active marrow with no excess of plasma cell types.

Treatment with prednisone and Endoxana was started in 1967. Radiographs in 1968 showed increase of deposits in the skull and left femur. A further course of radiotherapy was given. Now, twelve years after operation, the patient is seventy-three. The spine is stable without radiographic evidence of recurrence of tumour in the area of operation. She now complains of only mild discomfort in the lower part of the back.

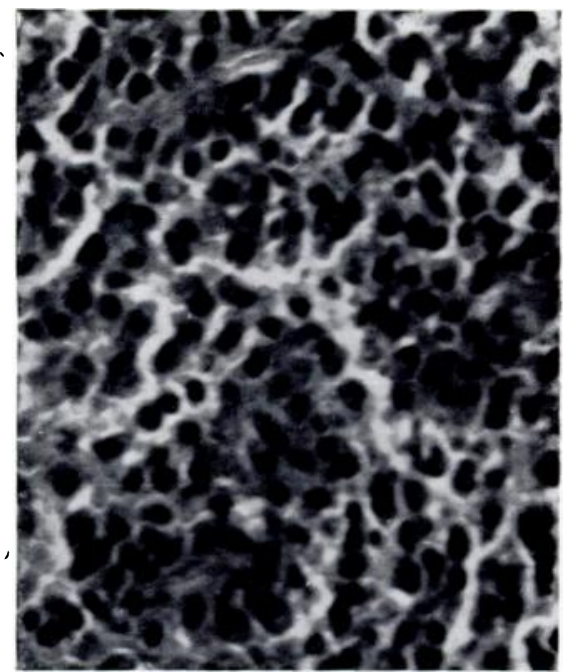

FIG. 3

Sheets of cells some showing slightly eccentric nuclei in basophilic cytoplasm of a myeloma. $(\times 360$.

Case 17-A boy aged twelve fell in 1954 and hurt his back. Radiographs at that time showed slightly increased density of the fourth lumbar vertebra. By 1957 he showed a severe rotational kyphoscoliosis and had developed disabling low back pain. He was immobilised in a plaster bed for four months. In 1958, because of increasing pain, the area was explored through an extraperitoneal approach. The body of the fourth lumbar vertebra was found to contain undifferentiated tissue. This was curetted

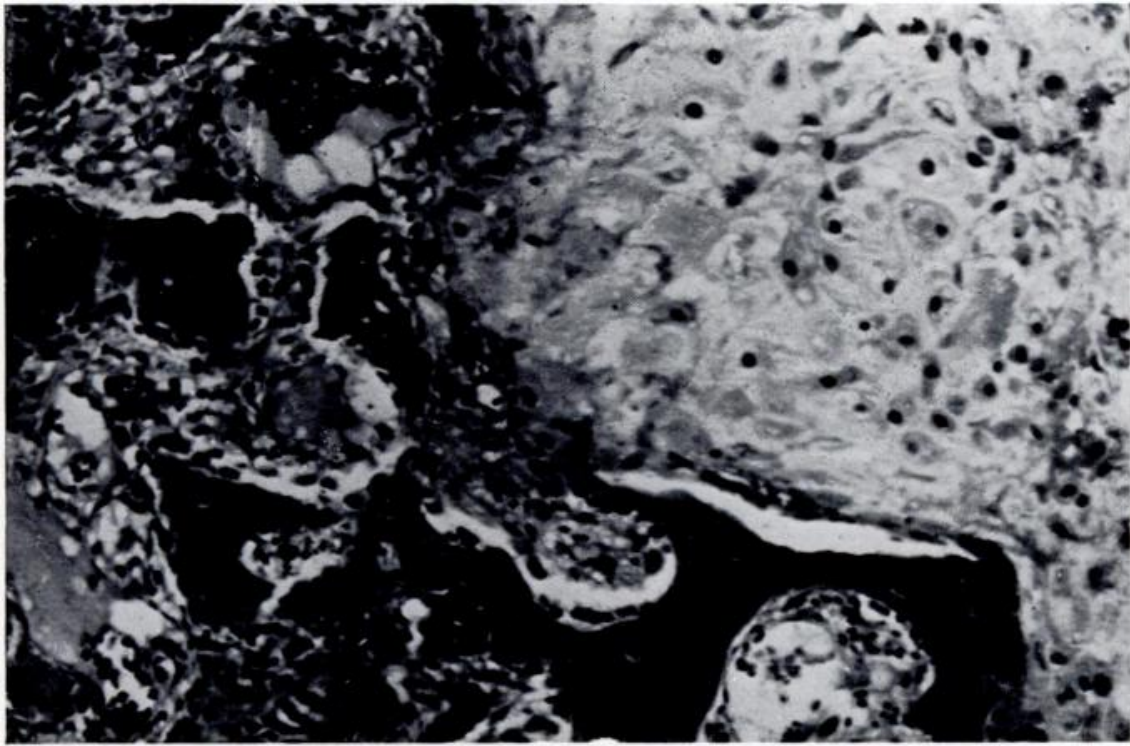

Fig. 4

Cellular cartilage with some atypical cells infiltrating irregularly among bone trabeculae. $(\times 180$. $)$

out. The curvature was corrected and wedged in place with autogenous bone. Histological examination of the material enabled a diagnosis of chondrosarcoma to be made (Fig. 4).

Progress after operation was uneventful. At the most recent review eleven years after operation, the patient was entirely well, and in full-time employment as a milkman. The only deformity is a slight rotational scoliosis shown on forward flexion.

vol. 52 B, NO. 2, MAY 1970

C 


\section{DISCUSSION}

Radical operation may not immediately commend itself in the treatment of malignant tumours of the spinal column, because in most cases it is unrealistic to think in terms of cure. In this series of localised lesions, improved prospects in terms of pain relief and delay in the development of neurological complications have made it clear that there is a place for laterally planned focal excision. In certain circumstances, although in a minority of patients, the

TABLE IV

Results of Treatment in Eighteen Patients

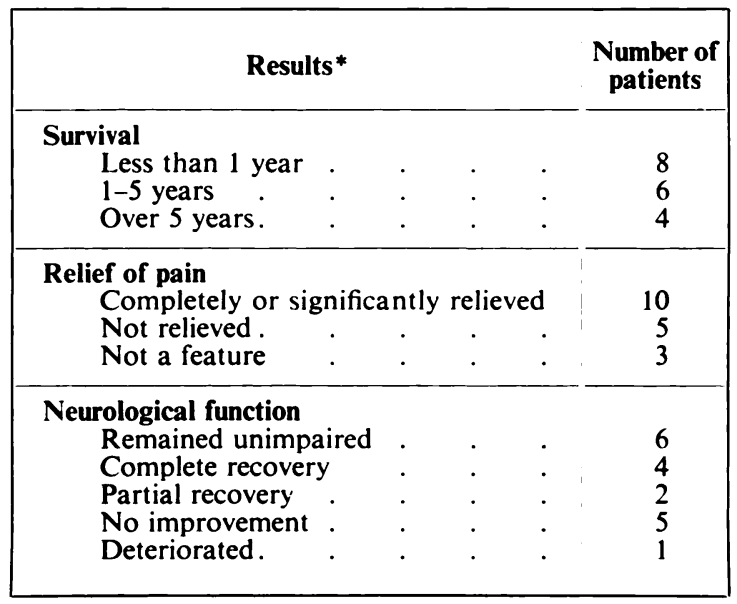

* The patient in Case 11 has not yet completed his first year after operation and is not included in these estimates of the results of treatment.

long-term results have far exceeded expectation. By such focal approaches it has been possible to arrive at a diagnosis when other methods of investigation have failed. By this method it has been possible to effect useful excision of the tumour as well as stabilisation by more effective anterior grafting techniques. Some patients would require surgical intervention irrespective of the precise diagnosis, because of local indications such as paraplegia, neurological deterioration, or extensive infiltration of a vertebra: in such patients there is no object in extensive clinical investigations or performing a separate biopsy by aspiration, because with a suitable approach the biopsy and appropriate surgical treatment can be combined in a single procedure.

Most authors have expressed dissatisfaction with laminectomy in the approach to lesions situated anteriorly. Bucy (1963) referred to the danger to the cord from sudden angulation produced by the collapse of vertebral body, and the difficulty of posterior fusion following decompressive laminectomy. He described the remarkable metal implant used by Kryaenbuhl to correct the deformity and support the vertebral column. Kennady and Stern (1962) referred to the "cuff" distribution of tumour material and the difficulty of removing the anterior portion by laminectomy. Their solution was to leave open the dura "to mitigate further symptoms from pressure of unremoved tumour anteriorly". Halnan and Roberts (1967) noted that decompression was most commonly effected by laminectomy, but suggested that "an anterior approach may often be better and leave the spine more stable". Brice and McKissock (1965) found that the relationship of the tumour mass to the spinal theca was a prognostic factor, the anteriorly placed or cuff lesions being of more sinister significance. In this respect they observed that surgical accessibility might be important. 
Whereas certain tumours such as secondaries from a well differentiated thyroid carcinoma, and solitary myeloma, offer a more favourable prognosis, the biopsy result is not always a reliable indication of prognosis. It is accepted that certain tumours-such as the metastatic deposits from a hypernephroma-are quite unpredictable in their response to radiotherapy, whereas others-such as chondrosarcoma-are positively radioresistant. In patients so affected early surgical treatment would be all-important in palliation. From the point of view of planning the treatment, the solitary nature of a tumour may be of great importance because it may imply a relatively slow rate of progression of the pathological process whatever its basic nature. Valderrama and Bullough (1968) mentioned this aspect of the prognosis in myeloma, noting that the individual host-tumour response, the nature of which is not clearly understood, was an unpredictable but possibly important influence on prognosis.

\section{SUMMARY}

1. A review of nineteen cases of malignant spinal tumour treated surgically is presented.

2. Four cases are presented in detail.

3. The results in terms of survival are not assessed, because the effect of operation on survival cannot be estimated in a small series without controls. However, if the patient does survive for a considerable time, the value of operation can be assessed in terms of its contribution to the quality of survival, in relieving pain or improving or protecting neurological function. 4. The limitations of laminectomy are compared with the possible advantages of anterior approaches.

We wish to thank colleagues in Musgrave Park Hospital, Belfast, for permission to study cases; Dr J. E. Morison for his help in the pathological interpretation and the preparation of photomicrographs, and Dr G. J. A. Edelstyn of the Northern Ireland Radiotherapy Centre for his help and advice. Our thanks are also due to Mr M. H. M. Harrison, Birmingham, for keeping us appraised of the progress of the patient in Case 8. This work was conducted with the aid of a grant from the Northern Ireland Hospitals Authority.

\section{REFERENCES}

Brice, J., and McKissock, W. (1965): Surgical Treatment of Malignant Extradural Spinal Tumours. British Medical Journal, 1, 1341.

BuCY, P. C. (1963): The Treatment of Malignant Tumours of the Spine. Neurology, 13, 938.

CAPENER, N. (1954): The Evolution of Lateral Rhachotomy. Journal of Bone and Joint Surgery, 36-B, 173.

Halnan, K. E., and Roberts, P. H. (1967): Paraplegia Caused by Spinal Metastasis from Thyroid Cancer. British Medical Journal, 3, 534.

Kennady, J. C., and Stern, W. E. (1962): Metastatic Neoplasms of the Vertebral Column Producing Compression of the Spinal Cord. American Journal of Surgery, 104, 155.

Mullan, J., and Evans, J. P. (1957): Neoplastic Disease of the Spinal Extradural Space. Archives of Surgery, 74, 900.

Valderrama, J. A. F., and Bullough, P. G. (1968): Solitary Myeloma of the Spine. Journal of Bone and Joint Surgery, 50-B, 82.

Young, J. M., and Funk, F. J. Jun. (1953): Incidence of Tumor Metastasis to the Lumbar Spine. Journal of Bone and Joint Surgery, 35-A, 55. 